\title{
MicroRNA-210 regulates cell proliferation and apoptosis by targeting regulator of differentiation 1 in glioblastoma cells
}

\author{
Shuai Zhang ${ }^{1}$, Niansheng Lai ${ }^{2}$, Keman Liao ${ }^{1}$, Jun Sun ${ }^{1}$, Yuchang Lin ${ }^{1}$ \\ ${ }^{1}$ Department of Neurosurgery, Wuxi Second Hospital Affiliated to Nanjing Medical University, Wuxi City, ${ }^{2}$ Department of Neurosurgery, \\ the First Affiliated Hospital of Wannan Medical College, Wuhu, China
}

\begin{abstract}
MicroRNAs (miRNAs) are small noncoding RNAs that negatively regulate protein biosynthesis and participate in the pathogenesis of various tumours. Previous studies have shown that miR-210 is highly expressed in different types of human cancers, including glioblastoma multiforme (GBM). However, the role that miR-210 plays in GBM remains unclear. Here, we detected the expression and examined the function of miRNA-210 in GBM cells. Furthermore, we investigated the possible molecular mechanisms by which miRNA-210 mediates cell proliferation and apoptosis. Fifteen GBM and five normal brain tissues, in addition to the U87MG and U251 GBM cell lines, were analysed in this study. We found that miR-210 was upregulated in GBM tissues and cell lines when compared to normal brain tissue. Cell counting and flow cytometric assay results demonstrated that upregulation of miR-210 induced cell proliferation and decreased cell apoptosis, respectively. In addition, downregulation of miR-210 inhibited cell proliferation and induced apoptosis. We also detected a miR-210 target, regulator of differentiation 1 (ROD1), which is involved in GBM progression. Knockdown of ROD 1 reversed the growth arrest and apoptosis that were originally induced by miR-210 inhibition. We propose that miR-210 regulates cell proliferation and apoptosis in GBM cells by targeting ROD 1. Our findings may provide a new potential therapeutic target for the treatment of GBM.
\end{abstract}

Key words: microRNA-210, glioblastoma, ROD1, proliferation, apoptosis.

\section{Introduction}

Glioblastoma multiforme (GBM) is the most common malignant brain tumour, accounting for $45.2 \%$ of all central nervous system tumours. Glioblastoma multiforme often infiltrates diffusely into normal tissue, making it difficult to completely eliminate via traditional surgery and radiotherapy. This leads to a poor outcome, with a five-year survival rate of less than $5 \%$ and an average survival time of only 10-14 months post-diagnosis $[11,16]$. Hence, it is import- ant to determine the mechanisms involved in the development of GBM and find more effective therapies to treat this deadly disease.

Research examining microRNAs (miRNAs) has grown exponentially since they were first discovered in 1993. MicroRNAs are non-coding RNAs that are 18-25 nucleotides in length, and they represent $1 \%$ to $2 \%$ of the eukaryotic transcriptome [2]. MiRNAs can lead to translational repression and/or mRNA degradation of their targets. Many miRNAs have 
been reported to be correlated with various carcinomas and have emerged as vital regulators in certain biological processes, including cell proliferation, migration, differentiation, apoptosis, and angiogenesis. For example, down-regulation of miR-150 inhibits cell proliferation and induces apoptosis in non-small-cell lung cancer (NSCLC) cells in culture. As another example, miR-27a suppression inhibits cell proliferation and migration by targeting mitogen activated protein kinase kinase 4 (MAP2K4) in human osteosarcoma cells $[6,12]$. These studies explored the expression and molecular mechanisms of miRNAs in specific tumour types, and determined that these small RNAs are novel biomarkers and therapeutic targets for anti-tumour treatments.

Previously, we found that miRNA-210, one of the most significantly upregulated miRNAs in hypoxic cells, is highly expressed in human gliomas and confers a poor prognosis in glioma patients [10]. Furthermore, an increase in miR-210 expression directly correlated with the histopathological grade of astrocytic tumours [9]. In the current study, we investigated the expression of miR-210 in GBM tissues and cell lines. Moreover, we explored its function in the regulation of proliferation and apoptosis, and determined that the regulator of differentiation 1 (ROD1), a protein also known as polypyrimidine trace binding protein 3 (PTBP3), is probably the target of miRNA-210 in GBM cells.

\section{Material and methods Tissue samples}

A total of 15 GBM tissues and five normal tissues were obtained from surgical resection at the Wuxi Second Hospital affiliated to Nanjing Medical University. Tissue samples from nine men and six women (mean age: $59.9 \pm 7.7$ years; age range: $46-72$ years) were included in the study. Pathological information was obtained after surgery and glioma stage and grade were determined based on the 2007 World Health Organization (WHO) Classification System. Five normal brain tissue samples were obtained from adult patients with craniocerebral injuries. A partial resection of brain tissue was required as decompression treatment to reduce intracranial pressure in these patients. Specimens were snap-frozen in liquid nitrogen and stored at $-80^{\circ} \mathrm{C}$. Patient permission was obtained for the collection and examination of all samples, and this study was approved by the Ethics Committee of Nanjing Medical University.

\section{Cell culture}

The U87MG and U251 cell lines were obtained from the Cell Resource Centre, Peking Union Medical College, which is the headquarters of the National Infrastructure of Cell Line Resources, National Science \& Technology Infrastructure (NSTI). The NSTI determined that the cell lines were free of mycoplasma contamination via polymerase chain reaction (PCR) analysis, and confirmed the species of origin for the two cell lines by PCR. All of the results from these tests can be viewed on the following website: http://cellresource.cn. Cells were grown in Dulbecco's modified Eagle's medium (DMEM; Hyclone, USA) supplemented with $10 \%$ foetal bovine serum (FBS; Gibco, USA) and $1 \%$ penicillin-streptomycin. Cells were incubated at $37^{\circ} \mathrm{C}$ in a saturated humidity atmosphere containing $5 \% \mathrm{CO}_{2}$. For all experiments, the cells were detached and one third of the cells were re-seeded in plates. All the cells used in this study were at passage 20 . The medium was changed on the cells every three days.

\section{Oligonucleotide transfection}

The miR-210 inhibitor and mimic and the ROD1 siRNA were chemically synthesised by Genepharma (Genepharma, China). The miR-210 inhibitor, a small, modified, single-stranded RNA molecule, was used to downregulate miR-210 expression, whereas the miR210 mimic, a small, modified, double-stranded RNA molecule, was used to upregulate miR-210 expression. The siRNA was utilised to block the expression of ROD1. Scramble oligonucleotide sequences were used as negative controls. Relative sequences were as follows: hsa-miR-210 inhibitor: 5'-UCAGCCGCUGUCACACGCACAG-3'; negative control oligonucleotide for the inhibitor: 5'-CAGUACUUUUGUGUAGUACAA-3'; hsa-miR-210mimic:5'-CUGUGCGUGUGACAGCGGCUGAAGCCGCUGUCACACGCACAGUU-3'; negative control oligonucleotide for the mimic: 5'-UUCUCCGAACGUGUCACGUTTACGUGACACGUUCGGAGAATT-3'. The transfection efficiency of the cells was identified via SYBR Green quantitative real-time reverse transcriptase polymerase chain reaction (qRT-PCR). Transfections were carried out using Lipofectamine 2000 (Invitrogen, USA) according to the manufacturer's protocol. 


\section{Total RNA isolation, reverse transcription, and quantitative real-time RT-PCR}

Total RNA, including miRNA, was extracted from the tissues or cells using Trizol reagent (Invitrogen) following the manufacturer's instructions. For ROD1, qRT-PCR was performed using ImProm-IITM reverse transcriptase (Promega, USA) and the QuantiFast SYBR Green PCR Kit (Qiagen, Germany). Detection was performed with the ABI7500 Real-time PCR system instrument (Applied Biosystems, USA). Relative primers were as follows: ROD1 forward: 5'-AAGGAAATGAATGGGCAGCCGTTAG-3'; ROD1 reverse: 5'-CATGTAGTTGAGGTCAATGAAGGGGTC-3; glyceraldehyde-3phosphate dehydrogenase (GAPDH) forward: $5^{\prime}$-GCTCTCTGCTCCTCCTGTTC-3'; GAPDH reverse: 5'-GACTCCGACCTTCACCTTCC-3'. Hairpin-it ${ }^{T M}$ miRNAs RT-PCR Quantitation Kit (Genepharma) was utilised to determine miR-210 expression, and the U6 small nuclear RNA (snRNA) Real-time RT-PCR Normalisation Kit (Genepharma) was used as the control.

The expression levels of ROD1 and miR-210 were calculated by the $2^{-\Delta \Delta C t}$ analysis method and were normalised to the controls.

\section{Western blot analysis}

The primary antibodies used in this study were anti-ROD1 (Santa Cruz, USA) and anti-GAPDH (Beyotime, China). Equal amounts of protein samples were mixed with equal amounts of sodium dodecyl sulphate (SDS) sample buffer and separated via $12 \%$ SDS-polyacrylamide gel electrophoresis (PAGE). Gels were run for one hour, and the separated proteins were transferred to polyvinylidene fluoride (PVDF) membranes. The membranes were then blocked with $5 \%$ nonfat powdered milk in tris-buffered saline with 10\% Tween-20 (TBST) for one hour. Membranes were incubated with primary antibody at $4^{\circ} \mathrm{C}$ overnight. Secondary antibody incubations were conducted using a goat anti-mouse antibody conjugated to horseradish peroxidase. Visualisation was performed using chemiluminescence.

\section{Cell proliferation assays}

Post-transfection, cells were seeded in 96-well plates at a density of 5000 cells per well for 12,24 , 36, 48, and 72 hours. Cell Count Kit-8 (CCK8; Dojindo, Japan) was used to measure cell proliferation according to the manufacturer's protocol. $10 \mu \mathrm{l}$ of
CCK8 solution was added into the medium of each well and incubated for 1.5 hours. The absorbance was read at $450 \mathrm{~nm}$ via a microplate system.

\section{Cell apoptosis assays}

Cells were seeded in six-well plates. After 48 hours the cells were washed with cold PBS and subsequently treated with Annexin $\mathrm{V}$ and propidium iodide (PI) using Alexa Fluor 488 annexin V/Dead Cell Apoptosis Kit (Invitrogen) to determine the cell apoptosis according to the protocol and then analysed by a flow cytometer (Beckman Coulter, USA).

\section{Statistical analysis}

All data were analysed using SPSS 11.0 for Windows (SPSS Inc., USA). Statistical significance was determined using the Student's $t$-test. Differences were considered significant when $p<0.05$.

\section{Results \\ Expression of miR-210 in glioblastoma multiforme tissues and cell lines}

To examine the expression of miR-210 in GBM tissue compared to normal tissue, we detected miR210 levels via qRT-PCR in GBM and normal brain tissue. Fifteen specimens diagnosed as GBM were examined, and five normal brain tissues were utilised as controls. In addition, miR-210 expression in the U87MG and U251 GBM cell lines was detected and compared to miR-210 expression in normal brain tissue. The expression levels were normalised to U6 RNA levels in the cells/tissues. As shown in Fig. 1A, miR-210 expression in the GBM tissues was significantly higher when compared to normal brain tissues $(p<0.001)$. Additionally, miR-210 expression in the U87MG and U251 GBM cell lines was higher than in normal brain tissue, as shown in Fig. 1B $(p<0.05)$.

\section{Upregulation of miR-210 promotes cell proliferation and inhibits cell apoptosis in glioblastoma multiforme cells}

To decipher the biological significance of miR-210 in GBM, the chemically synthesised miR-210 mimic and inhibitor were transfected into the U87MG and U251 GBM cell lines to upregulate and downregulate miR-210 expression, respectively. Transfection efficiency was evaluated via qRT-PCR and is shown in Fig. 2A and 2B. The U87MG cell line exhibited higher 
A

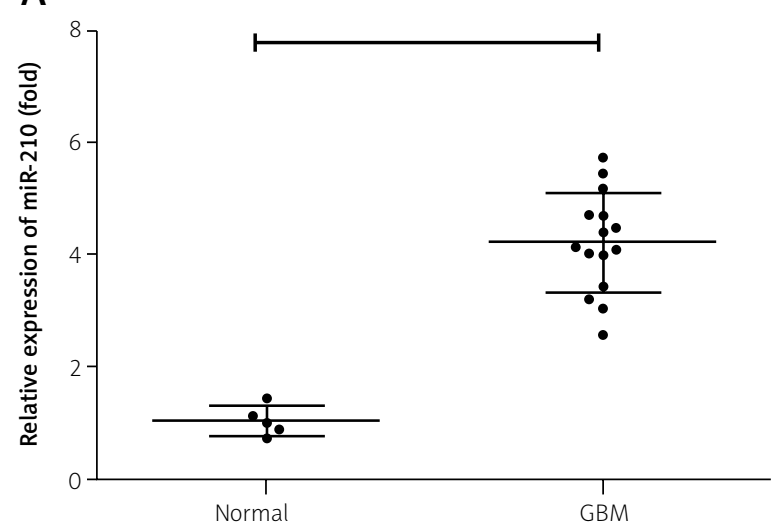

B

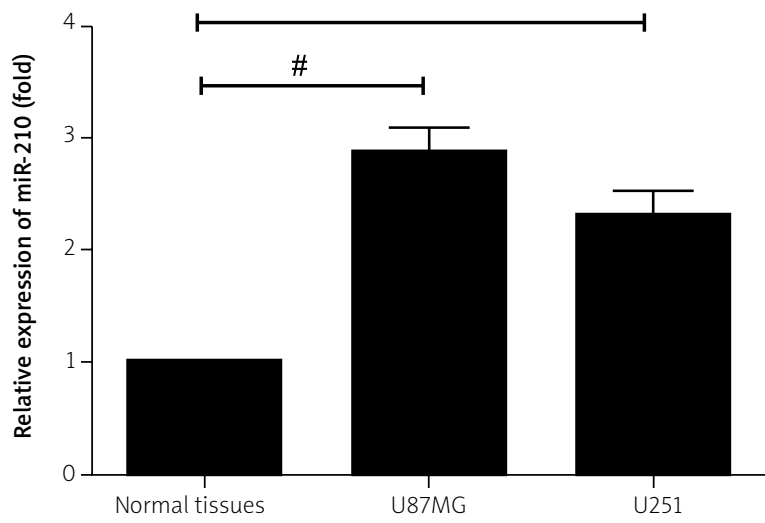

Fig. 1. A) Fifteen glioblastoma multiforme (GBM) and five normal brain tissue samples were utilised to examine miR-210 expression. Quantitative real-time reverse transcriptase PCR (qRT-PCR) was performed, and the relative expression between the groups is shown $\left({ }^{\#} p<0.05\right)$. B) The relative expression levels of miR-210 in the U87MG and U251 GBM cell lines compared to normal brain tissue were measured by qRT-PCR (\# $p<0.05)$.

transfection efficiency than U251 cells; therefore, all of our experiments in this study were conducted in the U87MG cell line. To investigate the effect miR-210 has on proliferation in GBM cells, we performed CCK8 assays on the transfected U87MG cells. These cell proliferation assays revealed that mimic-induced miR-210 overexpression led to a statistically significant increase in U87MG cell proliferation compared to the negative control cells (Fig. $2 \mathrm{C}, p<0.05$ ). Conversely, the proliferation rate of the U87MG cells treated with the miR-210 inhibitor was decreased when compared to control cells (Fig. 2D, $p<0.05$ ).

Next, we analysed apoptosis in the transfected GBM cells via flow cytometry. As shown in Fig. 3, U87MG cells transfected with the miR-210 inhibitor demonstrated a statistically significant increase in apoptotic rate when compared to controls, while the cells transfected with the miR-210 mimic exhibited a decrease in apoptosis $(p<0.05)$. Thus, miR-210 expression may be upregulated in GBM cells and tissues because expression of this microRNA promotes proliferation and regulates apoptosis in GBM cells.

\section{Regulator of differentiation 1$$
\text { as a potential target of miR-210 }
$$$$
\text { in glioblastoma multiforme }
$$

Because ROD1 has been identified as a miR-210 target in HEK293 cells, we next sought to investigate ROD1 as a potential miR-210 target in GBM cells. First, ROD1 expression was examined in GBM tissues at both the mRNA and protein levels. We found that expression of ROD1 was significantly lower in GBM tissues than in normal tissues (Fig. 4A, $p<0.001$ and Fig. 4B). To examine whether ROD1 is a likely target of miR-210 in GBM cells, we observed ROD1 expression in U87MG cells transfected with the miR-210 mimic or inhibitor. The miR-210 mimic effectively reduced ROD1 expression as detected by western blot analysis, whereas the miR-210 inhibitor led to an increase in ROD1 expression (Fig. 4C). This data indicates that ROD1 is probably a target of miR-210 in GBM cells.

\section{Knockdown of regulator \\ of differentiation 1 reverses the cell proliferation and apoptosis induced by miR-210}

Since we demonstrated that downregulation of miR-210 via a specific inhibitor could suppress cell proliferation and induce cell apoptosis in GBM cells (Fig. 2), we then wanted to determine whether ROD1 is directly involved in miR-210 regulation of GBM cell activity. Cell proliferation and apoptosis assays were performed on GBM cells transfected with a miR210 inhibitor or co-transfected with the miR-210 inhibitor and siRNA to knockdown ROD1. Regulator of differentiation 1 expression was remarkably reduced by the SiRNA as detected via western blot (Fig. 5A). Additionally, cell proliferation analysed by the CCK 8 assay demonstrated that the proliferation of GBM cells induced by miR-210 was abrogated by the knockdown of ROD1 (Fig. 5B, $p<0.001$ ). Furthermore, cell apoptosis analysis detected by flow 
A
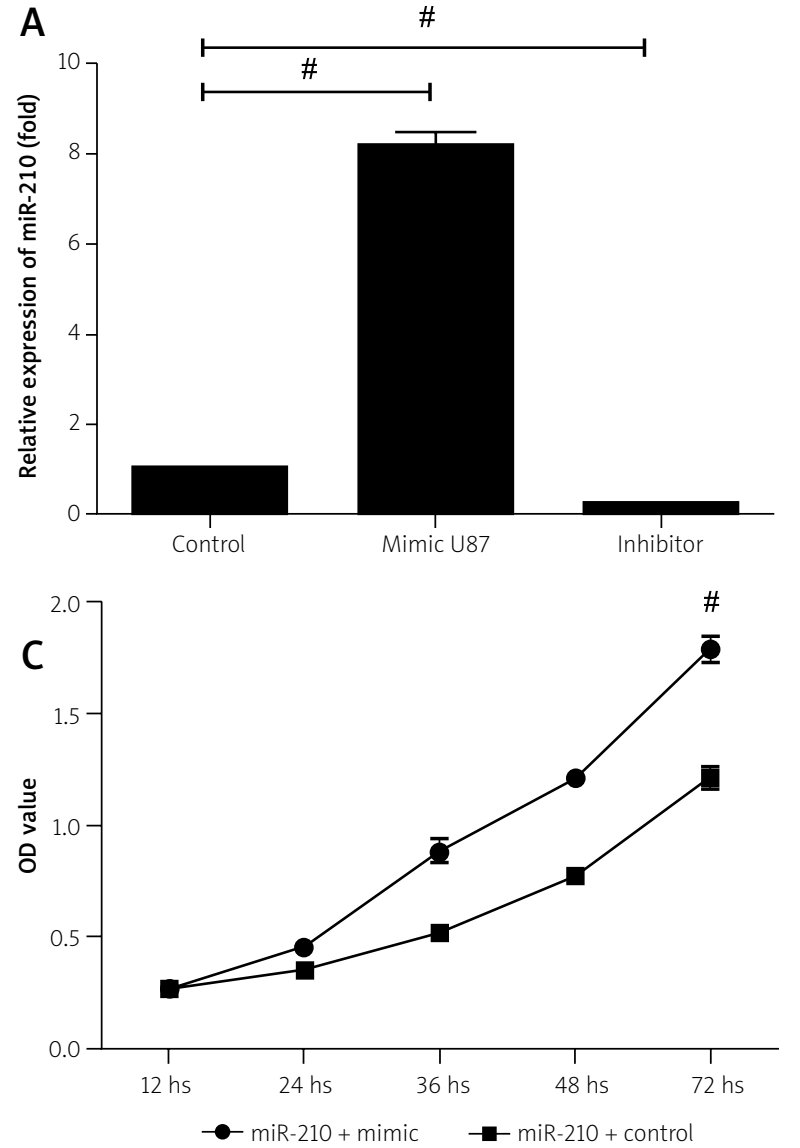

B
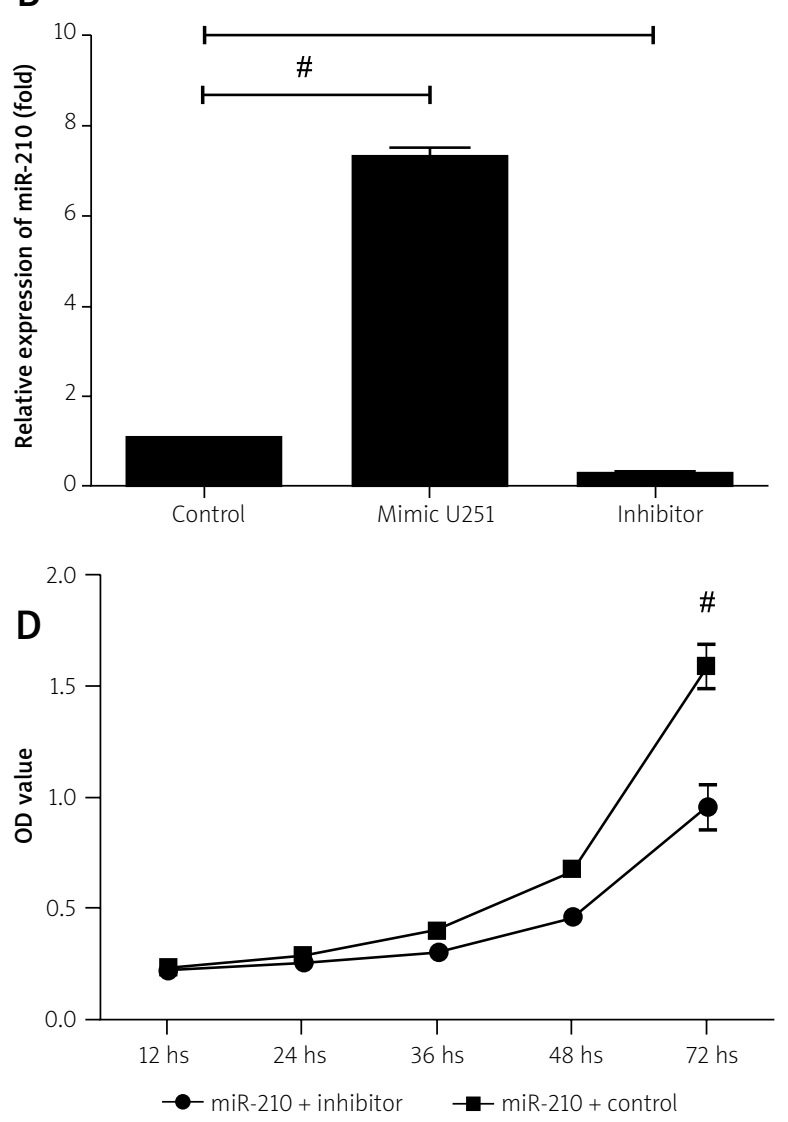

Fig. 2. A, B) The U87MG and U251 glioblastoma multiforme (GBM) cell lines were transfected with the miR-210 mimic or inhibitor. Forty-eight hours post-transfection, the cells were harvested and samples were prepared for quantitative real-time reverse transcriptase polymerase chain reaction (qRT-PCR). MiR-210 levels in the U87MG and U251 GBM cell lines were measured and compared to the non-transfected control group. MiR-210 was significantly upregulated in the cells expressing the mimic and significantly downregulated in the cells transfected with the inhibitor ( $\left.{ }^{*} p<0.05\right)$. The U87MG cells exhibited higher transfection efficiency than the U251 cells. C) Cell proliferation of U87MG cells transfected with the miR-210 mimic was measured by CCK8 analysis ( $\left.{ }^{\#} p<0.05\right)$. D) U87MG cells transfected with the miR-210 inhibitor were analysed for cell proliferation via CCK8 analysis ( $\left.{ }^{*} p<0.05\right)$.

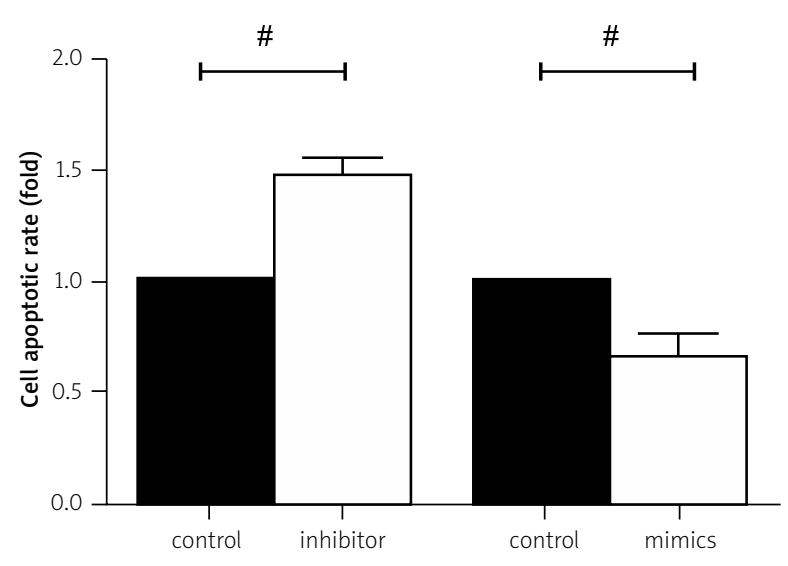

Fig. 3. After transfection with the miR-210 mimic or inhibitor, U87MG cell apoptosis was measured by flow cytometry $\left({ }^{\#} p<0.05\right)$. cytometry revealed that the decrease in apoptosis observed after miR-210 expression was abolished by the knockdown of ROD1 (Fig. 5C, $p<0.05$ ). The above results indicate that knockdown of ROD1 expression rescues the growth arrest and apoptosis that was observed when the GBM cells were treated with the miR-210 inhibitor.

\section{Discussion}

Accumulating studies have demonstrated that miRNAs play crucial roles in the occurrence and progression of malignant tumours. MiRNA dysregulation can be observed in diseased tissues or body fluids such as plasma, urine, tears, and cerebrospinal fluid [21]. Specific miRNA expression and/or levels of 

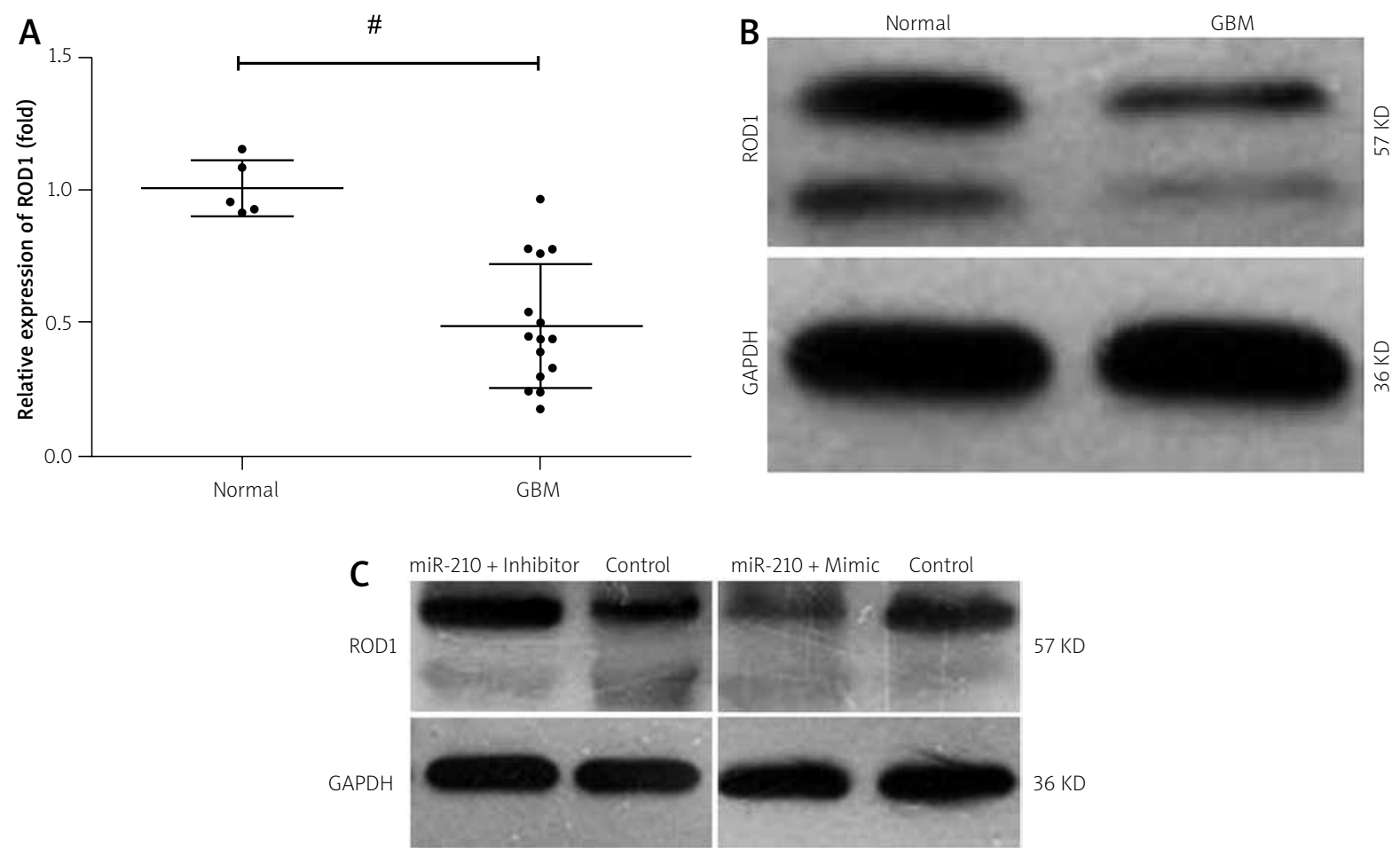

Fig. 4. A) Regulator of differentiation 1 (ROD1) expression was measured via real-time polymerase chain reaction (RT-PCR) in fifteen glioblastoma multiforme (GBM) and five normal brain tissue samples $\left({ }^{\#} p<0.05\right)$. B) Regulator of differentiation 1 protein expression in GBM and normal brain tissue was measured by western blot analysis. According to the instructions of the ROD1 antibody, the upper band is considered to be ROD1 with an apparent molecular weight of 57KD. C) U87MG cells were transfected with the miR-210 inhibitor or mimic and 48 hours post-transfection were analysed for ROD1 expression via immunoblot.

expression in particular tissues may predict certain diseases. Thus, these miRNAs can be selected as candidate biomarkers for the diagnosis and prognosis of various tumours $[20,22,24]$. The expression and function of various miRNAs have been reported in glioma $[17,25,26]$. In the current study, we determined the expression and function of miR-210 in GBM cells.

The stem-loop of miR-210 is located in an intron of a noncoding RNA that is transcribed from AK123483 on chromosome 11 p15.5 [13]. AK123483 is regarded as the pri-miR-210, which is then processed to the pre- and then the mature miR-210. Most studies conclude that miR-210 is a specific target of hypoxia inducible factor-1 (HIF-1), whereby HIF-1 directly binds to a hypoxia-responsive element (HRE) in the proximal miR-210 promoter. The HRE is responsible for the hypoxic induction of AK 123483 and may coordinate the expression of genes downstream of miR-210 [7]. MiR-210 is upregulated by hypoxia in multiple cell lines. Hypoxic conditions are also a common feature of the tumour microenviron- ment. MiR-210 expression is increased in most solid tumours, and its expression correlates with a negative prognosis [3]. MiR-210 has been shown to be involved in angiogenesis, DNA damage response, invasion, proliferation, apoptosis, and the cell cycle. Anton et al. [1] reported that miR-210 is upregulated in preeclamptic placentas, and that it regulates cell viability. MiR-210 overexpression was shown to reduce trophoblast invasion, while miR-210 inhibition promoted invasion. The authors concluded that miR-210 is a novel predictive serum biomarker for preeclampsia. Wang et al. [18] reported expression of miR-210 in neural progenitor cells, whereby they identified Bcl-2 adenovirus E1B 19 kDa-interacting protein 3 (BNIP3), which is regulated by HIF-1 $\alpha$ and promotes cell death, as a direct functional target of miR-210. MiR-210 directly suppressed BNIP3 expression, which subsequently reduced cell death.

Our previous report showed that oligodendroglial tumours exhibit a significantly reduced level of miR-210 as compared to normal brain tissue. In 

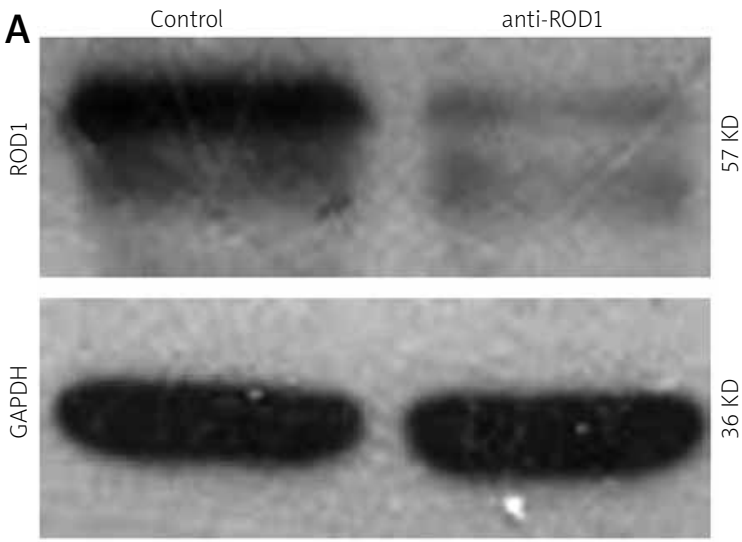

C

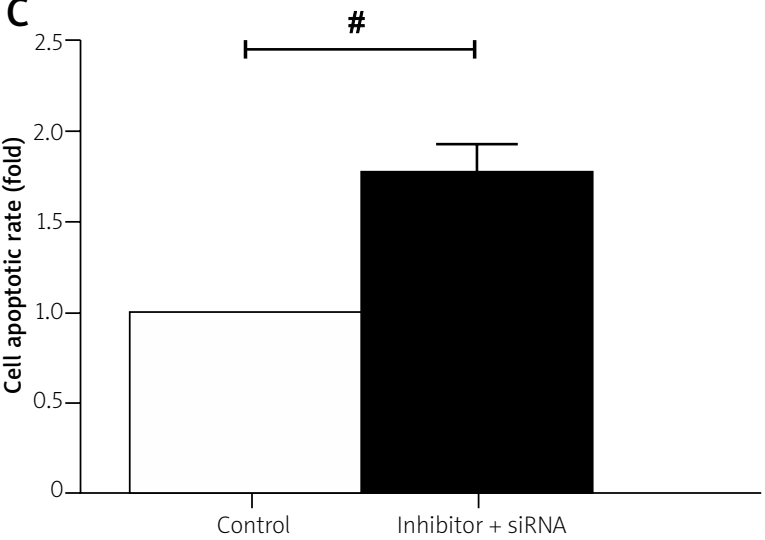

contrast, astrocytic tumours demonstrate significantly increased levels of miR-210. Furthermore, the expression of miR-210 positively correlates with the grade of astrocytic tumours. Thus, miR-210 could be a novel candidate biomarker for glioma diagnosis and prognosis $[9,10]$. In the study presented here, we determined that miR-210 expression is increased in GBM tissue and cells when compared to normal brain tissue, and that miR-210 could potentially modulate tumour progress by affecting cell growth and viability. Our finding that miR-210 is upregulated in GBM could have enhanced significance since recent studies have shown that miRNAs expressed in the brain have relatively short half-lives and can be rapidly degraded $[8,14,19]$. Functional analyses were performed to examine the affect that miR-210 expression has on proliferation and apoptosis in GBM cells. CCK8 cell proliferation analysis and flow cytometric assays examining apoptosis indicated that introduction of a miR-210 inhibitor into GBM cells significantly suppressed cell number and induced apoptosis, respectively. Moreover, transfection of the miR-210 mimic significantly promoted cell growth.

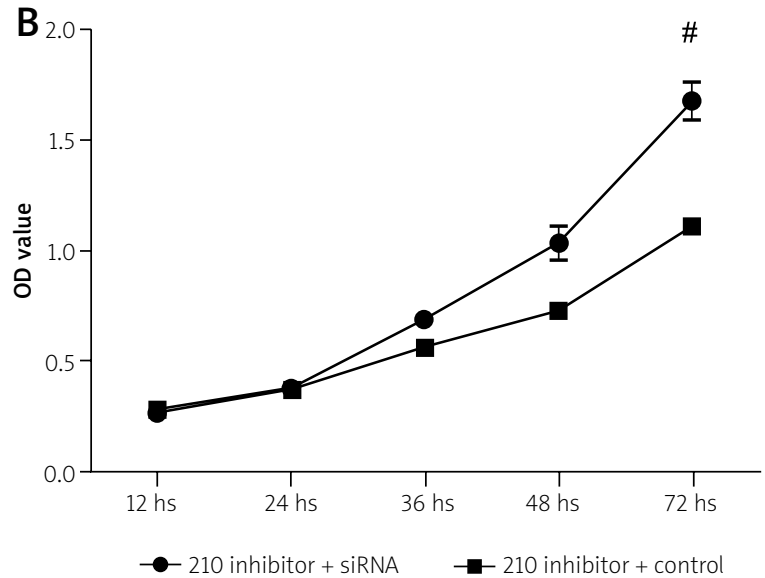

Fig. 5. A) U87MG cells were transfected with regulator of differentiation 1 (ROD1) siRNA, and subsequently ROD1 expression was analysed by western blot. B) After transfection with the miR-210 inhibitor or the miR-210 inhibitor plus ROD1 siRNA, U87MG cell proliferation was measured with the CCK8 assay (\# $p<0.05)$. C) After transfection with the miR-210 inhibitor or with the miR-210 inhibitor and the ROD1 SiRNA, U87MG cell apoptosis was measured via flow cytometry $\left({ }^{\#} p<0.05\right)$.

These findings suggest that miR-210 plays a critical role in the proliferation and apoptosis potential of GBM cells.

We then explored ROD1, also known as PTBP3, as a potential target of miR-210 in GBM cells. ROD1 has been confirmed as a direct but seedless target of miR-210 in HEK293 cells [4]. Immuno-precipitation analysis performed by Fasanaro et al. [5] identified ROD1 as a miR-210-seedless transcript enriched in miR-210-containing RNA-induced silencing complexes. In both the presence and absence of hypoxic conditions, expression of ROD1 inhibited cell proliferation and induced cell apoptosis. This same study also found two well-established miR-210 targets, ephrin-A3 and RAD52 homolog, indicating the reliability of the experiment. Furthermore, Rna22 prediction, thermodynamic stability analysis, and luciferase activity all indicated that ROD1 is a direct target of miR-210 in HEK293 cells. Taken together with our findings in GBM, it is likely that miR-210 directly targets ROD1 in GBM and could affect the biological activity of GBM cells. ROD1, a member of the PTBP family of proteins [15], is a repressive splicing regula- 
tor expressed in various cell types. Regulator of differentiation 1 was reported to be primarily expressed in haematopoietic cells in rats [23], thus the role that ROD1 plays in differentiation was examined in mammalian cells. Overexpression of ROD1 was found to inhibit 12-0-tetradecanoylphorbol-13-acetate (TPA)induced megakaryocytic differentiation and sodium butyrate-induced erythroid differentiation in the pluripotent human hematopoietic leukaemia cell line K562 [23]. Our research demonstrated that ROD1 is expressed in human brain tissues, and may play an important role in the development of GBM via regulation by miR-210. Our results show that overexpression of miR-210 decreased ROD1 levels; and ROD1 inhibition abolishes the anti-tumour effects observed in GBM cells treated with the miR-210 inhibitor. Thus, miR-210 could be a candidate biomarker and an important factor in GBM through the targeting ROD1.

In summary, we demonstrated that miR-210 is overexpressed in GBM tissues, and that miR-210 may function as an oncogene in GBM cells. Based on our observations and previous findings by other groups, we conclude that miR-210 could be a novel biomarker for GBM, and it probably regulates tumour progression through the targeting of ROD1. Since GBM is a form of cancer that progresses and spreads quickly, traditional therapies such as surgery and chemoradiotherapy have only limited effectiveness in treating this aggressive disease. Therefore, new approaches such as molecular targets would be highly beneficial in the therapeutic treatment of GBM. Targeting miR-210 may provide a new therapeutic option in the treatment of this deadly disease.

\section{Acknowledgments}

This study was supported by the Joint Research of Wuxi Medical Management Centre TGZXL1309.

\section{Disclosure}

Authors report no conflict of interest.

\section{References}

1. Anton L, Olarerin-George AO, Schwartz N, Srinivas S, Bastek J, Hogenesch JB, Elovitz MA. miR-210 inhibits trophoblast invasion and is a serum biomarker for preeclampsia. Am J Pathol 2013; 183: 1437-1445.

2. Dalmay T, Edwards DR. MicroRNAs and the hallmarks of cancer. Oncogene 2006; 25: 6170-6175.
3. Fasanaro P, D’Alessandra Y, Di Stefano V, Melchionna R, Romani S, Pompilio G, Capogrossi MC, Martelli F. MicroRNA-210 modulates endothelial cell response to hypoxia and inhibits the receptor tyrosine kinase ligand Ephrin-A3. J Biol Chem 2008; 283: 15878-15883.

4. Fasanaro P, Greco S, Lorenzi M, Pescatori M, Brioschi M, Kulshreshtha R, Banfi C, Stubbs A, Calin GA, Ivan M, Capogrossi MC, Martelli F. An integrated approach for experimental target identification of hypoxia-induced miR-210. J Biol Chem 2009; 284: 35134-35143.

5. Fasanaro P, Romani S, Voellenkle C, Maimone B, Capogrossi MC, Martelli F. ROD1 is a seedless target gene of hypoxia-induced miR-210. PLoS One 2012; 7: e44651.

6. Gu XY, Wang J, Luo YZ, Du Q, Li RR, Shi H, Yu TP. Down-regulation of miR-150 induces cell proliferation inhibition and apoptosis in non-small-cell lung cancer by targeting BAK1 in vitro. Tumour Biol 2014; 35: 5287-5293.

7. Huang X, Le QT, Giaccia AJ. MiR-210 - micromanager of the hypoxia pathway. Trends Mol Med 2010; 16: 230-237.

8. Krol J, Busskamp V, Markiewicz I, Stadler MB, Ribi S, Richter J, Duebel J, Bicker S, Fehling HJ, Schubeler D, Oertner TG, Schratt G, Bibel M, Roska B, Filipowicz W. Characterizing light-regulated retinal microRNAs reveals rapid turnover as a common property of neuronal microRNAs. Cell 2010; 141: 618-631.

9. Lai N, Zhu H, Chen Y, Zhang S, Zhao X, Lin Y. Differential expression of microRNA-210 in gliomas of variable cell origin and correlation between increased expression levels and disease progression in astrocytic tumours. Folia Neuropathol 2014; 52: 79-85.

10. Lai NS, Dong QS, Ding H, Miao ZL, Lin YC. MicroRNA-210 overexpression predicts poorer prognosis in glioma patients. J Clin Neurosci 2014; 21: 755-760.

11. Ostrom QT, Gittleman H, Farah P, Ondracek A, Chen Y, Wolinsky Y, Stroup NE, Kruchko C, Barnholtz-Sloan JS. CBTRUS statistical report: Primary brain and central nervous system tumors diagnosed in the United States in 2006-2010. Neuro Oncol 2013; 15 Suppl 2: ii1-56.

12. Pan W, Wang H, Jianwei R, Ye Z. MicroRNA-27a Promotes Proliferation, Migration and Invasion by Targeting MAP2K4 in Human Osteosarcoma Cells. Cell Physiol Biochem 2014; 33 : 402-412.

13. Saini HK, Griffiths-Jones S, Enright AJ. Genomic analysis of human microRNA transcripts. Proc Natl Acad Sci U S A 2007; 104: 17719-17724.

14. Sethi P, Lukiw WJ. Micro-RNA abundance and stability in human brain: specific alterations in Alzheimer's disease temporal lobe neocortex. Neurosci Lett 2009; 459: 100-104.

15. Spellman R, Llorian M, Smith CW. Crossregulation and functional redundancy between the splicing regulator PTB and its paralogs nPTB and ROD1. Mol Cell 2007; 27: 420-434.

16. Van Meir EG, Hadjipanayis CG, Norden AD, Shu HK, Wen PY, Olson JJ. Exciting new advances in neuro-oncology: the avenue to a cure for malignant glioma. CA Cancer J Clin 2010; 60: 166-193.

17. Wan X, Cheng Q, Peng R, Ma Z, Chen Z, Cao Y, Jiang B. ROCK1, a novel target of miR-145, promotes glioma cell invasion. Mol Med Rep 2014; 9: 1877-1882.

18. Wang F, Xiong L, Huang X, Zhao T, Wu LY, Liu ZH, Ding X, Liu S, Wu Y, Zhao Y, Wu K, Zhu LL, Fan M. miR-210 suppresses BNIP3 to 
protect against the apoptosis of neural progenitor cells. Stem Cell Res 2013; 11: 657-667.

19. Wang H, Chiu M, Xie Z, Chiu M, Liu Z, Chen P, Liu S, Byrd JC, Muthusamy N, Garzon R, Croce CM, Marcucci G, Chan KK. Synthetic microRNA cassette dosing: pharmacokinetics, tissue distribution and bioactivity. Mol Pharm 2012; 9: 1638-1644.

20. Wang X, Meng X, Li H, Liu W, Shen S, Gao Z. MicroRNA-25 expression level is an independent prognostic factor in epithelial ovarian cancer. Clin Transl Oncol 2014; 16: 954-958.

21. Weber JA, Baxter DH, Zhang S, Huang DY, Huang KH, Lee MJ, Galas DJ, Wang K. The microRNA spectrum in 12 body fluids. Clin Chem 2010; 56: 1733-1741.

22. Wu CW, Ng SC, Dong Y, Tian L, Ng SS, Leung WW, Law WT, Yau TO, Chan FK, Sung JJ, Yu J. Identification of microRNA-135b in stool as a potential noninvasive biomarker for colorectal cancer and adenoma. Clin Cancer Res 2014; 20: 2994-3002.

23. Yamamoto H, Tsukahara K, Kanaoka Y, Jinno S, Okayama H. Isolation of a mammalian homologue of a fission yeast differentiation regulator. Mol Cell Biol 1999; 19: 3829-3841.

24. Zhao J, Lu Q, Zhu J, Fu J, Chen YX. Prognostic value of miR-96 in patients with acute myeloid leukemia. Diagn Pathol 2014; 9: 76.

25. Zhong Q, Wang T, Lu P, Zhang R, Zou J, Yuan S. miR-193b promotes cell proliferation by targeting Smad3 in human glioma. J Neurosci Res 2014; 92: 619-626.

26. Zhou J, Wang W, Gao Z, Peng X, Chen X, Chen W, Xu W, Xu H, Lin MC, Jiang S. MicroRNA-155 promotes glioma cell proliferation via the regulation of MXI1. PLoS One 2013; 8: e83055. 